\title{
On the Existence of Codes with Constant Bounded PMEPR for Multicarrier Signals ${ }^{1}$
}

\author{
Masoud Sharif, and Babak Hassibi \\ Department of Electrical Engineering \\ California Institute of Technology, Pasadena, CA 91125 \\ \{masoud, hassibi\}osystems.caltech.edu
}

\begin{abstract}
It has been shown that with probability one the peak to mean envelope power ratio (PMEPR) of any random codeword chosen from a symmetric QAM/PSK constellation is $\log n$ where $n$ is the number of subcarriers [1]. In this paper, the existence of codes with nonzero rate and PMEPR bounded by a constant is established.
\end{abstract}

\section{INTRODUCTION}

Recently, Paterson and Tarokh have raised the question of what is the trade off between rate, minimum distance, and PMEPR of a code [2]. It is also proved that the VarsharmovGilbert upper bound remains the same for spherical codes with PMEPR less than $8 \log n$ for large $n$. In [1], based on the asymptotic analysis of PMEPR, it is shown that the PMEPR of spherical codes and symmetric QAM/PSK constellations is $\log n$. Therefore, it is not surprising to have no trade off between rate and PMEPR as long as the PMEPR is less than $\log n$ with probability one. However, without any contradiction with the result of [1], there still might be exponentially many codewords with constant PMEPR, even though the probability of randomly choosing one of them goes to zero, and henceforth, they are rare!

The normalized complex envelope of a multicarrier signal with $n$ subcarriers may be represented as $s_{C}(\theta)=\sum_{i=1}^{n} c_{i} e^{j \theta i}$ where $0 \leq \theta<2 \pi$ and $C=\left(c_{1}, \ldots, c_{n}\right)$ is the complex modulating vector with entries from a given complex constellation $\mathcal{Q}$. The admissible modulating vectors are called codewords and the ensemble of all possible codewords constitute the code $\mathcal{C}$. Then, the PMEPR of each codeword $C \in \mathcal{C}$ is defined as,

$$
\operatorname{PMEPR}_{\mathcal{C}}(C)=\max _{0 \leq \theta<2 \pi} \frac{\left|s_{C}(\theta)\right|^{2}}{E\left\{\|C\|^{2}\right\}}
$$

Similarly, PMEPR $R_{\mathcal{C}}$ is defined as the maximum of Eq. (1) over all codewords in $\mathcal{C}$. Clearly, when $\mathcal{C}$ is a random code such that the $c_{i}$ 's are chosen independently from a constellation with average power $E_{a v}$, the average power of $C$ is $n E_{a v}$. Whenever we drop $\mathcal{C}$ from PMEPR, we mean $\mathcal{C}$ is a random code with average power $n E_{a v}$.

\section{Peak Reduction By Choosing Signs}

Given the codeword $C=\left(c_{1}, \ldots, c_{n}\right)$, we consider the design of an optimum sign, $\epsilon_{i}$, for each subcarrier, in order to minimize the PMEPR of the resulting codeword $C_{\epsilon}=$ $\left(\epsilon_{1} c_{1}, \ldots, \epsilon_{n} c_{n}\right)$. Clearly the worst case PMEPR of a codeword $C$ is of the order of $n$. We also know from the result of

\footnotetext{
${ }^{1}$ This work was supported in part by the National Science Foundation under grant no. CCR-0133818, by the office of Naval Research under grant no. NO0014-02-1-0578, and by Caltech's Lee Center for Advanced Networking.
}

[1], that a randomly chosen vector $C$ will have PMEPR of $\log n$ for large values of $n$, and therefore randomly choosing signs should work well for large $n$. This raises the question of how much further reduction in PMEPR can we get by choosing the best sign for each subcarrier? Since changing signs does not affect the average power, we can focus on minimizing the peak of $s_{C}(\theta)$ over the $\epsilon_{i}$ 's. Here is the statement of the problem: For any given complex vector $C=\left(c_{1}, \ldots, c_{n}\right)$ where $\left|c_{i}\right| \leq \sqrt{E_{\max }}$, consider the following minimization problem:

$$
\min _{e} \max _{0 \leq \theta \leq 2 \pi}\left|\sum_{i=1}^{n} \epsilon_{i} c_{i} e^{j \theta i}\right|
$$

where $\epsilon=\left(\epsilon_{1}, \ldots, \epsilon_{n}\right)$ and $\epsilon_{i} \in\{+1,-1\}$.

- What is the best achievable value of (2) for any codeword $C$ ?

- How can we design the optimum vector $\epsilon$ ?

\section{Codes with Constant Bounded PMEPR}

In this section, we answer the first question raised above.

Theorem 1. Suppose $c_{i}$ 's are chosen from a constellation $\mathcal{Q}$ with maximum and average power $E_{m a x}$ and $E_{a v}$, respectively. Then, there exists a sign vector $\epsilon=\left(\epsilon_{1}, \ldots, \epsilon_{n}\right)$ such that $\operatorname{PMEPR}\left(C_{\epsilon}\right) \leq k E_{m a x} / E_{a v}$, for sufficiently large $n$, where $C_{\epsilon}=\left(\epsilon_{1} c_{1}, \ldots, \epsilon_{n} c_{n}\right)$ and $k$ is a constant independent of $n$.

The proof of Theorem 1 is based on the result of [4] on bounded linear forms.

Corollary 1. For any q-ary symmetric constellation in which both $A$ and $-A$ are in the constellation, there exists a code with rate $R \geq 1-\log _{q} 2$ and with PMEPR less than a constant, for sufficiently large $n$.

Addressing the second question raised in section II, an algorithm to design the signs is proposed in [3]. The algorithm guarantees the PMEPR to be less than $c \log n$ for any $n$.

It is also worth mentioning that the approach in this paper can be used to study the peak to average power in multiple antenna systems using LD codes.

\section{REFERENCES}

[1] M. Sharif and B. Hassibi, "On multicarrier signals where the PMEPR of a random codeword is asymptotically $\log n$," $s u b$ mitted to IEEE Trans. Info., 2003.

[2] K. G. Paterson and V. Tarokh, "On the existence and construction of good codes with low peak to average power ratios," IEEE Trans. Info., vol. 46, no. 6, pp. 1974-1986, Sep. 2001.

[3] M. Sharif and B. Hassibi, "A deterministic algorithm that achieves the PMEPR of $c \log n$ for multicarrier signals," to appear in ICASSP 2003.

[4] J. Spencer, "Six standard deviations suffice," Trans. Amer. Math. Soc., vol. 289, no. 2, pp. 679-706, June 1985. 